\title{
Ermittlung der Kosten für Forschung und Lehre am UniversitätsSpital Zürich
}

\author{
Zur Ermittlung der Kosten für Forschung und Lehre hat das UniversitätsSpital Zürich \\ eine breit abgestützte Tätigkeitsanalyse durchgeführt. Die Daten zeigen einen An- \\ teil von $\mathbf{8 , 6 \%}$ an den Gesamtkosten. Dieser ist bedeutend tiefer als der von der \\ Preisüberwachung geforderte, empirisch nicht hergeleitete Normabzug von $23 \%$.
}

Hugo Keunea,

David Weibel ${ }^{b, c}$,

Bartholomäus Wissmath ${ }^{b, c}$

a UniversitätsSpital Zürich Direktor Finanzen

b w hoch $2 \mathrm{GmbH}$, Bern

c Dr. phil. Universität Bern, Institut für Psychologie Abteilung für Kognitive Psychologie, Wahrnehmung und Methodenlehre
Korrespondenz:

Hugo Keune

UniversitätsSpital Zürich

Direktion Finanzen

Rämistrasse 100

CH-8091 Zürich

Tel. 0442553465

hugo.keune[at]usz.ch

\section{Einleitung}

Das KVG sieht vor, dass die Kosten für universitäre Lehre und für Forschung nicht in den Tarifen für stationäre Behandlungen (Baserate) enthalten sein dürfen (vgl. Art. 49 Abs. 3 lit. b). Die Kosten seien daher, soweit in den Kostenträgern «stationäre Leistungen KVG» berücksichtigt, in Abzug zu bringen.

Was mit universitärer Forschung und Lehre gemeint ist, wurde vom Bundesrat im Rahmen einer Motion geklärt [1]: Die Forschung und universitäre Lehre umfasst demnach die Grundlagenforschung, die klinische Forschung, die Kosten für die ärztliche Ausbildung bis zum Staatsexamen (Studium) und die Kosten für die ärztliche Weiterbildung von Assistenzärzten bis zu deren Erwerb des Facharzt-Titels, jedoch ohne die Personalkosten für Assistenzärzte.

Die Ermittlung der Kosten für Forschung und Lehre ist für die Universitätsspitäler für die Festsetzung der stationären Tarife von grosser Bedeutung. Können die effektiven Kosten für universitäre Forschung und Lehre nicht erfasst werden, ist gemäss Empfehlung der Gesundheitsdirektorenkonferenz subsidiär ein Normabzug von maximal 12\% der gesamten Nettobetriebskosten vorzunehmen. Die Preisüberwachung und die Krankenversicherer werfen den Universitätsspitälern vor, über keine hinreichend genauen Kostenerhebungen zu verfügen und nehmen daher einen pauschalen Abzug von 23\% vor. Dieser Normabzug wird in keiner Art und Weise empirisch und datengestützt hergeleitet.

Die Ausscheidung der Kosten für Forschung und Lehre ist anspruchsvoll. Die Verbindung zu Leistungen in der medizinischen Versorgung ist eng. Es sind häufig dieselben Personen, die mit denselben Geräten in denselben Räumen unter Verwendung derselben Medikamente sowohl Versorgung wie auch Forschung und Lehre betreiben. Methodologisch stellt die Erfassung der Tätigkeitsanteile und damit der Kosten für Lehre und Forschung an Universitätsspitälern somit eine Herausforderung dar [2]. Das Bundesamt für Statistik (BFS) hat eine Vorgehensweise erarbeitet, wie dieser Herausforderung begegnet werden kann

\section{Evaluation des coûts de la}

\section{recherche et de l'enseignement}

\section{à l'Hôpital universitaire de Zurich}

Pour évaluer les coûts de la recherche et de l'enseignement, I'Hôpital universitaire de Zurich (USZ) a procédé à une vaste analyse de son activité. A cet effet, un ensemble de mesures a été mis en œuvre afin de garantir la validité, la fiabilité et l'objectivité de cette analyse. Les différentes composantes de l'activité ont été recensées au moyen d'une enquête en ligne menée de façon individuelle et rétrospective auprès de l'ensemble du corps médical, des académiciens ainsi que du personnel médico-thérapeutique et médico-technique. Les résultats montrent que la part de la recherche et de l'enseignement à I'USZ s'élève à environ $15 \%$, toutes personnes interrogées confondues. En pondérant ce chiffre par le taux d'occupation et en le multipliant par le salaire, on obtient un total de $\mathbf{9 7 , 9}$ millions de francs pour la recherche et l'enseignement, ce qui correspond à $8,6 \%$ des coûts globaux. Soit un taux nettement plus bas que celui de $23 \%$ exigé par le Surveillant des prix et dénué de tout fondement empirique.

[3]. Gemäss BFS ist diese Methode «in sich vollständig und geschlossen (...) und entspricht der wissenschaftlichen Herangehensweise, welche zur Erstellung von beschreibenden Statistiken angewendet wird» (S. 34). Konkret empfiehlt das BFS folgendes Vorgehen:

- Direkte Befragung der Mitarbeitenden

- Bottom-up-Vorgehensweise im Sinne einer Vollerhebung 
- Hohe Standardisierung

- Schriftliche und retrospektive Befragung

- Online-Befragung mittels Erhebungssoftware

- Berücksichtigung der bezahlten und der unbezahlten Arbeitszeit

Im Rahmen der hier vorgestellten Erhebung am UniversitätsSpital Zürich (USZ) konnten alle diese Empfehlungen umgesetzt werden. Zudem wurden zusätzliche Massnahmen zur Qualitätssicherung getroffen, um hohe Validität, Reliabilität und Objektivität sicherzustellen. In mehreren empirisch fundierten Schritten konnte so eine Erhebungsmethodik entwickelt werden, die den Aufwand für die Bereiche medizinische Versorgung, studentische universitäre Ausbildung, ärztliche Weiterbildung zur Erlangung eines FMH-Titels sowie Forschung akkurat quantifiziert.

Basierend auf dieser Methode wurden am USZ 2012 und 2013 die Tätigkeitsanteile für die Ärzteschaft und Akademiker(innen) sowie das medizinisch-therapeutische und medizinisch-technische (MTT) Personal im Rahmen einer Vollerhebung ermittelt. Die Tätigkeitsanteile in den vier Bereichen konnten dabei über eine online verfügbare Software im Sinne einer Selbstdeklaration individuell und retrospektiv angegeben werden. Auf die Darstellung der Erhebung für die Berufsgruppen Pflege und Administration, die ebenfalls durchgeführt wurde, wird an dieser Stelle aus Platzgründen verzichtet. Auch beziehen sich die hier präsentierten Resultate auf die Tätigkeitsanalyse 2013 .

\section{«Dieser Normabzug wird in keiner Art und Weise empirisch und datengestützt hergeleitet.»}

\section{Methode}

\section{Definition von Versorgung, Ausbildung, Weiter- bildung und Forschung}

Akkurate Werte der Tätigkeitsanteile können nur resultieren, wenn die Güte der Definition der Bereiche gegeben ist. Um dies sicherzustellen, wurde zunächst ein Think-Aloud-Test zur Überprüfung einer vom BFS entworfenen Definition [3] durchgeführt ( $\mathrm{n}=$ 20). So entstand eine prägnante Globaldefinition der vier Bereiche, die ergänzt wird durch eine Liste von einzelnen Tätigkeiten.

Weiter fand ein objektiver Definitionstest statt (n = 126). Hierbei wurden die Definitionen der vier Bereiche auf einer Skala beurteilt ( $1=$ unverständlich; $10=$ verständlich). Die Definitionen wurden als verständlich beurteilt, was sich an hohen Mittelwerten zeigt $(\mathrm{M}=7,6$ bis 8,$58 ; \mathrm{SD}=1,90$ bis 2,42$)$. Zur Be- stimmung der Eindeutigkeit mussten die Testpersonen zudem 77 konkrete Tätigkeiten zu den Posten Versorgung, Ausbildung, Weiterbildung und Forschung zuordnen. Die Resultate zeigen, dass die Zuordnungen in den meisten Fällen der A-priori-Klassifikation entsprechen. Weiter konnte ermittelt werden, dass die Liste der Tätigkeiten vollständig ist.

Abschliessend wurden die Definitionen in einer ärztlichen und einer MTT-Expertengruppe finalisiert. So resultierte für die Ärzteschaft und Akademiker(innen) sowie das MTT-Personal je eine verständliche, eindeutige und vollständige Definition. In der MTT-Definition wurde auf den Bereich der ärztlichen Weiterbildung verzichtet, da Tätigkeiten in diesem Bereich gemäss der Befragungen vernachlässigbar sind.

\section{Tagebuchstudie zur Überprüfung der Reliabilität} Eine heterogene Stichprobe $(n=47)$, die Mitarbeitende von 36 Kliniken umfasste, hat während des Zeitraumes von 15 Arbeitstagen (je nach Arbeitssituation 3 bis 10 Wochen) ein Online-Tagebuch bezüglich des Arbeitsaufwandes in den vier Tätigkeitsbereichen geführt. Danach wurde der Aufwand zusätzlich über die Zeitdauer der Tagebuchstudie in den vier Bereichen retrospektiv geschätzt. Zur Quantifizierung der Zuverlässigkeit der retrospektiven Schätzung wurde die durchschnittliche tägliche Schätzung mit der retrospektiven Ex-post-Schätzung korreliert. Die Korrelationen sind sehr hoch ausgefallen:

$$
\begin{array}{ll}
- & \mathrm{r}_{\text {Versorgung }}=0,91, \mathrm{p}<0,01 \\
- & \mathrm{r}_{\text {Ausbildung }}=0,82, \mathrm{p}<0,01 \\
- & \mathrm{r}_{\text {Weiterbildung }}=0,87, \mathrm{p}<0,01 \\
- & \mathrm{r}_{\text {Forschung }}=0,94, \mathrm{p}<0,01
\end{array}
$$

Die starken Zusammenhänge belegen, dass die Mitarbeitenden den jeweiligen Arbeitsaufwand für die vier Bereiche retrospektiv sehr gut abschätzen können. Dies zeigt, dass mit der Tätigkeitsanalyse zuverlässige Daten gewonnen werden können.

\section{Durchführung der Tätigkeitsanalyse}

Die online durchgeführte Tätigkeitsanalyse der Ärzteschaft, der Akademiker(innen) sowie des MTT-Personals fand zwischen Juni und August 2013 statt. Nach einer Vorinformation durch die Klinikleitung wurde ein persönlicher Zugangslink gesendet. Dieser verschaffte verschlüsselt den Zugriff auf das Tool, in dem nach der Auseinandersetzung mit der Definition der jeweilige Anteil der Arbeitstätigkeit für Versorgung, Ausbildung, Weiterbildung und Forschung rückblickend für 12 Monate erfasst werden konnte. Diese Angaben bezogen sich auf die bezahlte Arbeitszeit. Zusätzlich konnte die unbezahlte Arbeit für die Bereiche deklariert werden.

Auf diesem Wege wurden die Daten von 1193 Ärztinnen, Ärzten und Akademiker(inne)n sowie 636 MTT-Mitarbeitenden erhoben, was einem Rücklauf von $99 \%$ entspricht. Das Ziel einer Vollerhebung 
konnte somit erreicht werden. Die Erhebung lief partiell anonym ab: Den Mitarbeitenden wurde zugesichert, dass die Vorgesetzten nur Daten über verschiedene organisationale Einheiten hinweg erhalten (Werte nach Klinik), aber keine individuellen Daten.

\section{Abbildung 1}

Anteilsmässiger Aufwand der Ärzteschaft, der Akademiker(innen) und der MTT-Mitarbeitenden des USZ. (Bei der Ärzteschaft und den Akademiker(inne)n wurden für den Total-Wert die Bereiche Versorgung und Weiterbildung zusammengerechnet.)

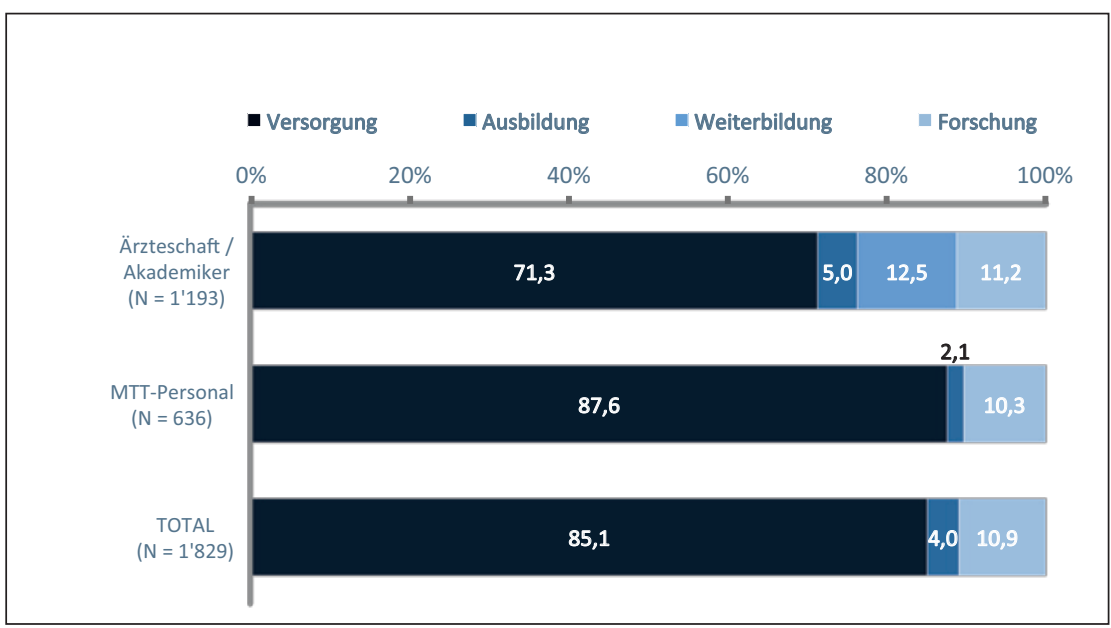

Abbildung 2

Anteilsmässiger Aufwand nach Tätigkeit.

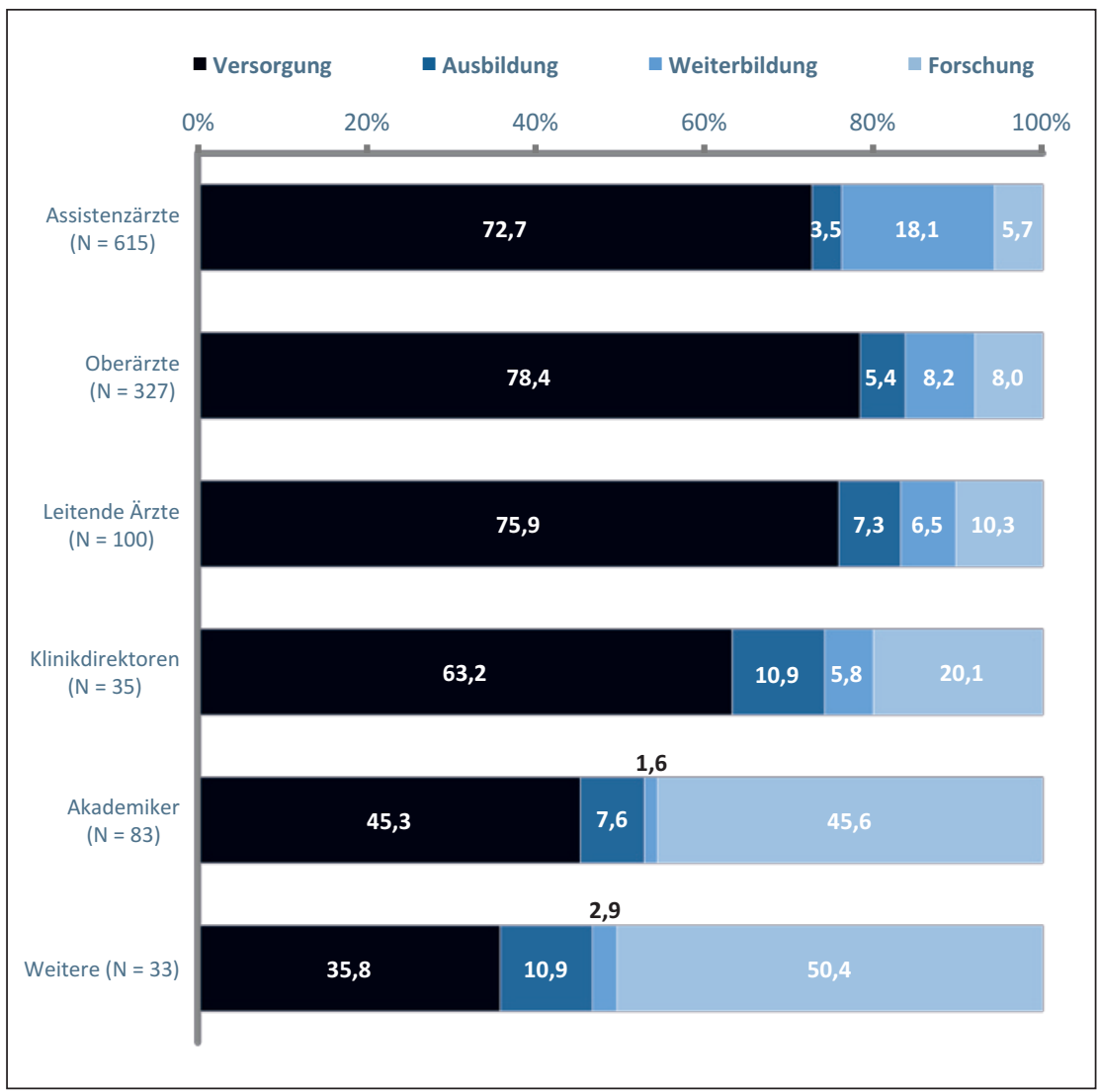

\section{Kosten}

Basierend auf der Tätigkeitsanalyse können die Personalkosten ermittelt werden. Bei der Tätigkeitsanalyse wurden sowohl die Tätigkeitsanteile der bezahlten Arbeitszeit am USZ (inkl. Überzeiten) wie auch die geleisteten Stunden in der Freizeit erfragt. Letztere wurden nicht bewertet und nicht in die Kosten einbezogen.

Für die Ermittlung der Kosten wurden die in der Tätigkeitsanalyse erhobenen Anteile der bezahlten Arbeitszeit mit dem Beschäftigungsgrad gewichtet und mit dem effektiven Lohn multipliziert.

Zur Bestimmung der gesamten Kosten im Bereich Forschung und Lehre mussten zu den Personalkosten noch die übrigen Kostenblöcke hinzugerechnet werden. Diese wurden analog den Vorgaben für die Versorgung gemäss den Branchenrichtlinien REKOLE von H+ im Rahmen der Kostenrechnung ermittelt. Sachkosten wurden direkt auf die Kostenstellen für Forschung und Lehre verbucht. Die Infrastruktur-, Kapital- und Overheadkosten wurden mittels Umlagen zugerechnet. Dabei kamen die jeweiligen Umlageschlüssel (Raumdatenbank, Anlagebuchhaltung, Anteil Vollzeitstellen etc.) zum Tragen. Die Kostenrechnung des USZ ist seit 2011 nach den Richtlinien von REKOLE zertifiziert und erfüllt damit die strengsten Vorgaben bezüglich Qualität und Transparenz.

\section{Resultate}

Abbildung 1 zeigt die ungewichteten Gesamtresultate. Insgesamt resultierte ein Versorgungsanteil von 85,1\% bei einem Anteil Forschung und Lehre (bzw. Ausbildung) von $14,9 \%$. Bei der Ärzteschaft und den Akademiker(inne)n wurden 83,8\% der Arbeit für Tätigkeiten für die Versorgung oder Weiterbildung aufgewendet. Der Anteil von Lehre und Forschung liegt bei 16,2\%. Für das MTT-Personal resultierte ein tieferer Anteil für Lehre und Forschung von 12,4\%.

Abbildung 2 zeigt die Werte der Ärzteschaft und Akademiker(innen) aufgeschlüsselt nach Tätigkeit. Diese zeigen, dass der Anteil Forschung und Lehre bei den leitenden Ärzt(inn)en höher ist als bei den Oberärzt(inn)en. Am tiefsten ist der Anteil bei den Assistenzärzten, am höchsten bei den Akademiker(inne)n.

Werden die erhobenen Tätigkeitsanteile mit dem Beschäftigungsgrad gewichtet und dem Lohn multipliziert, ergeben sich im Bereich der Forschung und Lehre 2013 Gesamtkosten von 97,9 Millionen Franken. Dies entspricht 8,6\% der Gesamtkosten.

\section{Diskussion}

Im Rahmen der umfassend validierten Tätigkeitsanalyse am USZ konnte empirisch belegt werden, dass der Tätigkeitsanteil Lehre und Forschung am USZ bei rund $15 \%$ liegt. Gewichtet nach dem Beschäftigungsgrad und multipliziert mit dem Lohn, ergibt dies Gesamtkosten für Forschung und Lehre 
von 97,9 Millionen Franken, was 8,6\% der Gesamtkosten ausmacht. Die Preisüberwachung und die Krankenversicherer fordern demgegenüber einen in keiner Weise hergeleiteten Normabzug von 23\%.

Die Güte der hier vorgestellten Erhebungsmethode ist insgesamt als hoch einzuschätzen, wie der nachfolgenden Auflistung zu entnehmen ist:
Innerhalb der Berufsgruppen wurden identische Fragebogen verwendet. Der Einsatz standardisierter Instruktionen und Erhebungsinstrumente gewährleistet grösstmögliche Standardisierung und Objektivität.

- Unabhängigkeit und partielle Anonymität: Die gesamte Deklaration erfolgte für die Teilnehmen-

\section{«Es bleibt zu hoffen, dass das Bundesverwaltungsgericht im Rahmen des laufenden Tariffestsetzungsverfahrens diese Tatsachen entsprechend würdigt.»}

- Hohe Validität der verwendeten Definitionen: In zahlreichen Interviews und einem objektiven Test konnte gezeigt werden, dass die Definitionen verständlich, eindeutig und vollständig sind.

- Hohe Reliabilität der retrospektiven Schätzungen: Die Ergebnisse der Tagebuchstudie legen nahe, dass akkurate retrospektive Schätzungen möglich sind und dass die erhobenen Daten keinen bedeutsamen oder systematischen Verzerrungen unterliegen. Nach Abschluss der Deklaration wurden zudem Personen mit besonders auffälligen Werten einem Interview unterzogen. Dabei konnten die Daten mit Hilfe von Dienstplänen und individuellen Erklärungen vollständig plausibilisiert werden.

- Hohe Datenqualität: Die Vollständigkeit der Daten konnte in diesem Projekt gewährleistet werden. Mit 99\% wurde ein sehr hoher Rücklauf erzielt, der den Rücklauf vergleichbarer Projekte übertrifft (vgl. [3]).

- Vollständige Standardisierung: Die Deklaration der Arbeitsanteile erfolgte online und schriftlich und wie vom BFS vorgeschlagen durch die Mitarbeitenden selbst (keine Vorgesetztenbefragung).

\section{Interaktiver Artikel}

Wollen Sie diesen Artikel kommentieren? Nutzen Sie dafür die Kommentarfunktion in der OnlineVersion oder sehen Sie nach, was Ihre Kolleginnen und Kollegen bereits geschrieben haben: www.saez.ch/aktuelle-ausgabe/interaktive-beitraege/ den erkennbar ausserhalb des direkten Zugriffs des USZ. Den Mitarbeitenden wurde weiter zugesichert, dass die Vorgesetzten nur Daten über verschiedene organisationale Einheiten hinweg erhalten.

Im Rahmen der Tätigkeitsanalyse am USZ wurden verschiedenste Massnahmen getroffen, um Validität, Reliabilität und Objektivität des Verfahrens sicherzustellen. Es kann daher davon ausgegangen werden, dass die Daten belastbar sind und die erhobenen Werte den tatsächlichen Tätigkeitsanteilen entsprechen. Angesichts der hohen Verlässlichkeit bei der Kostenermittlung mutet der Vorschlag der Preisüberwachung und der Krankenversicherer geradezu willkürlich an. Im Gegensatz zu der hier dargestellten Tätigkeitsanalyse ist der vorgeschlagene Normabzug nicht empirisch fundiert. Es bleibt zu hoffen, dass das Bundesverwaltungsgericht im Rahmen des laufenden Tariffestsetzungsverfahrens diese Tatsachen entsprechend würdigt.

\section{Literatur}

1 Cassis I. Motion 08.3847: Schaffung finanzieller Anreize für Spitäler, die Assistenzärztinnen und -ärzte weiterbilden; 2008.

2 Ott R. Grenzen und Lösungsansätze einer Kostenzuordnung auf Forschung, Lehre und Krankenversorgung in Universitätsklinika. Bayerisches Staatsinstitut für Hochschulforschung und Hochschulplanung; 2003.

3 Salgado-Thalmann E, Teotino T, Füglister A. Kosten der akademischen Lehre und Forschung in den Universitätsspitälern. Bundesamt für Statistik BFS; 2010 . 